\section{WEBWATCH}

\section{A model resource}

David Wilkinson's review in this issue reminds us that pattern formation is a central concept to the study of neural development. While empirica observations have begun to elucidate patterning

mechanisms, the theoretical analysis of pattern formation also has a long tradition. Hans Meinhardt and Alfred Gierer have advocated this approach since the early 1970 s, and they have collected an impressive array of mathematical models to support their ideas. These models can be found on their web site, 'Theoretical aspects of pattern formation and neuronal development'. The site, in Meinhardt's words, ".. is addressed primarily for experimental scientists, hoping to convince them that an integration of the theoretical and the experimental approach can provide a deeper understanding of what is going on. Emphasis is placed on the self-organizing aspect of development and on the generation of patterns starting from near-uniform tissues and cells".

The site uses animated computer simulations to illustrate a variety of aspects of pattern formation, from the establishment of the

embryonic axes to the pigmentation of the mollusc shell. There is plenty to interest the neuroscientist, with sections on axon guidance, growth cone navigation and the roles of boundaries as organizing regions. Admittedly, some of the animations are hard to follow because they run very fast, and they would benefit from some additional labelling, but otherwise this is a very useful resource.

The site is clearly written, and provides an accessible introduction to an aspect of developmental biology that scientists often dismiss as being too 'difficult'. Meinhardt recognizes that many biologists have an aversion to mathematics, and he hopes that this web site will help to encourage them to join in with his theoretical approach.

NEUROIMAGING

\title{
Searching for the baseline
}

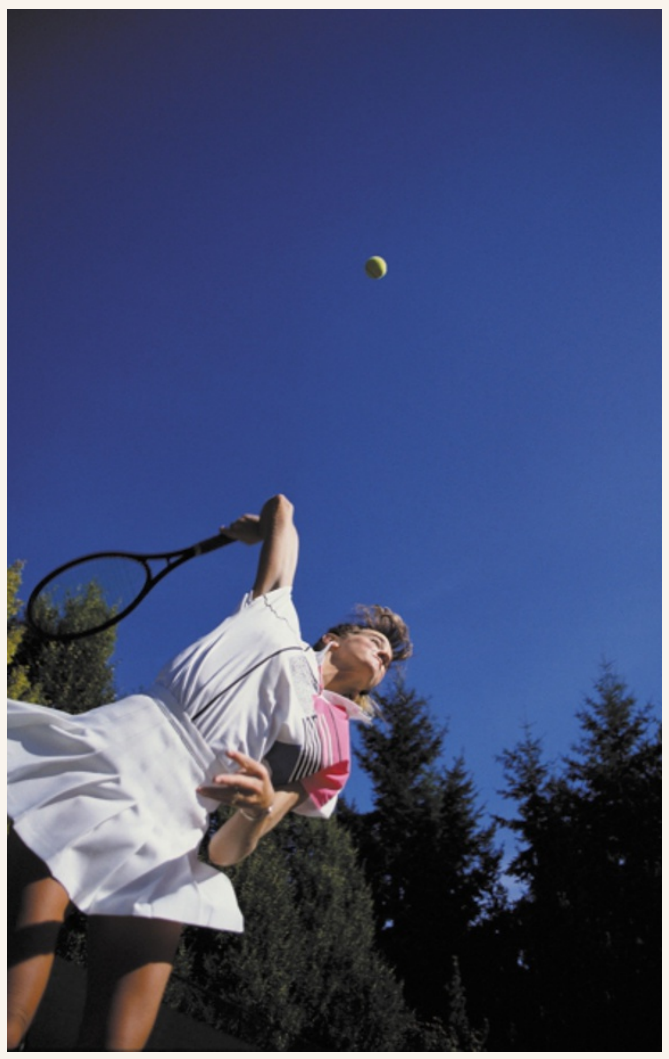

A fundamental aspect of scientific experimentation is the identification of a control or baseline state against which the condition of interest can be compared. Functional brain imaging studies that use positron emission tomography (PET) or functional magnetic resonance imaging are not an exception to this basic rule. In imaging studies, task-induced increases in regional brain activity during specific goal-directed behaviour are commonly observed by comparing between a specific experimental task and a control task. The difference between the two tasks is usually considered to represent the brain activity that is associated with the process of interest. This difference becomes manifest as a regionally specific and taskdependent increase in brain activity.

Although recording increases in brain activity is common practice, task-induced decreases in regional activity have also been observed even when the control task consists of lying quietly with eyes closed or passively viewing a stimulus. Interestingly, these decreases in brain activity frequently seem to be taskindependent, with little variation in location across a wide variety of tasks. What do these decreases in brain activation represent? Are they simply unrecognized increases in activation that are present only in the 'control' state? By this account, the 'control' state is nothing less than just another task with its own activation patterns. Or do such decreases in brain activity represent decreases from a true baseline state? This issue centres on the question of what is the baseline state of the brain.

Marcus Raichle and colleagues have used PET to measure a variety of metabolic and circulatory relationships between blood flow and oxygen consumption in the brain. The key measure in their studies was the oxygen extraction fraction (OEF) the fraction of the oxygen available to the brain that is used by the brain. They found a remarkable spatial uniformity in the OEF across the nervous system when measured in the resting state. This observation led them to propose that this measure might define a baseline state of brain activity.

None of the areas that are regularly observed to show decreases in activation during goal-directed behaviour showed an OEF that was significantly different from the hemispheric mean OEF. This suggests that these brain areas were not activated in the resting state. However, areas within extrastriate visual cortex did exhibit a significant increase in OEF relative to the hemispheric mean, suggesting that these areas are deactivated in the resting state. This interpretation is supported by the observation that areas of extrastriate cortex increase their blood flow when the eyes were opened, suggesting that the baseline state in these regions is observed when the eyes are open rather than closed.

The authors propose that the baseline state can be measured as the OEF and, therefore, decreases in brain activation represent true deflections from a baseline state rather than a return to baseline from an unrecognized increase in the control state. So, these areas might temporarily suspend functions that are specific to the baseline state during this period. Although more work is needed to determine unequivocally the meaning of these findings, they might have profound implications for functional neuroimaging and for our understanding of stimulus processing within the brain. This paper and two others by the same group that appear in the same issue of Proceedings of the National Academy of Sciences begin the exploration of this new territory.

\section{Peter Collins}

D) References and links

ORIGINAL RESEARCH PAPER Raichle, M. E. et al. A default mode of brain function. Proc. Natl Acad. Sci. USA 98, 676-682 (2001)

FURTHER READING Simpson, J. R. Jr et al. Emotion-induced changes in human medial prefrontal cortex: I. During cognitive task performance. Proc Natl Acad. Sci. USA 98, 683-687 (2001) | Simpson, J. R. Jr et al. Emotioninduced changes in human medial prefrontal cortex: II. During anticipatory anxiety Proc. Natl Acad. Sci. USA 98, 688-693 (2001)

WEB SITE Marcus Raichle's lab

ENCYCLOPEDIA OF LIFE SCIENCES Brain imaging: localization of brain functions | Brain imaging: observing ongoing neural activity 